\title{
A Method to Discriminate Between the Candida stellata and Saccharomyces cerevisiae in Mixed Fermentation on WLD and Lysine Agar Media
}

\author{
S. Di Maio ${ }^{1}$, G. Polizzotto ${ }^{1,2}$, D. Planeta ${ }^{2 *}$, D. Oliva ${ }^{1}$ \\ (1) Istituto Regionale della Vite e del Vino (IRVV), Regione Siciliana, Via Libertà 66, 90143 Palermo, Italy \\ (2) Dipartimento di Ingegneria e Tecnologie Agro-Forestali - Sezione Industrie - Università degli Studi di Palermo, Viale Scienze n. \\ 11, 90128 Palermo, Italy
}

Submitted for publication: June 2010

Accepted for publication: August 2010

Key words: Cycloheximide, Candida stellata, Saccharomyces cerevisiae, mixed fermentation

\begin{abstract}
This paper presents a simple method to distinguish between Candida stellata and Saccharomyces cerevisiae yeasts during microbiological analyses. The method is based on differential yeast growth on a medium containing cycloheximide and a medium containing lysine as only nitrogen source (lysine agar). The cycloheximide resistance of 45 yeast strains belonging to Candida stellata, Hanseniaspora uvarum, Hanseniaspora guilliermondii, Metschnikowia pulcherrima, Torulaspora delbrueckii, Zygosaccharomyces bailii, Kluyveromyces thermotolerans and Zygoascus hellenicus, and 14 strains of Saccharomyces cerevisiae and Saccharomyces bayanus on WL nutrient agar, was assayed. Cycloheximide resistance is characteristic of the species $H$. uvarum, $H$. guilliermondii and $Z$. hellenicus, while for the other yeasts it depends on the strain and the concentration of cycloheximide used. Two $\mathrm{mg} / \mathrm{L}$ of cycloheximide allows selective counting of a strain of $C$. stellata (Cs3) compared to one of the sensitive $S$. cerevisiae strain (NDA21). Similar results can be obtained on lysine agar, but counts are reliable only with the additional spreading of a monolayer of Saccharomyces cells. The different cycloheximide resistance of $C$. stellata and $S$. cerevisiae can be used in the microbiological analysis of mixed cultures to monitor the individual growth of the two yeast species. This method can be applied to the study of mixed fermentations with other non-Saccharomyces species. The modified use of lysine agar is useful to a certain extent in the distinction of multistarter yeasts from the indigenous yeasts.
\end{abstract}

\section{INTRODUCTION}

In recent years, many researchers have focused their attention on studying the potential oenological use of non-Saccharomyces yeasts that are naturally present in musts and that are capable of producing secondary compounds that influence the organoleptic quality of wine as a result of their peculiar metabolic activities (Fleet, 2003; Romano et al., 2003). The use of some non-Saccharomyces yeasts was also investigated in experimental winemaking and in some cases the results are so encouraging that consideration for their application in oenology is increasing. Analysing the ability of the indigenous yeasts to carry out pure fermentation of the Macabeo variety Spanish grape, Clemente-Jimenez et al. (2004) identified strains of Candida stellata that were able to achieve complete fermentation, leaving little residual sugar and producing ethanol typical of table wines. They also showed that strains of Pichia fermentans and Hanseniaspora uvarum were able to produce large quantities of glycerol, even if distinguished by lower fermenting activity. The same authors also described wines characterised by a well-balanced composition of higher alcohol, also produced with fermentation by $H$. uvarum, Issatchenkia orientalis and C. stellata strains. Higher glycerol quantities have been produced in wines fermented by $C$. stellata, but with lower fermentation activity of the strains and an abundance of produced acetaldehyde, acetoin and higher alcohols (Ciani \& Ferraro, 1996).

However, the reduced fermentation activity widespread among these non-Saccharomyces yeasts and the excessive production of off-flavour compounds do not permit the use of mostnon-Saccharomyces yeasts as a starter in pure fermentations. A new trend in oenological experimentation foresees nonSaccharomyces/Saccharomyces mixed inocula in must, with promising results (Jolly et al., 2006). Mixed preparations of Kluyveromyces thermotolerans and Saccharomyces cerevisiae (www.chr-hansen.com) and Torulaspora delbruekii, $K$. thermotolerans and S. cerevisiae (www.chr-hansen.com) are already on sale for the production of wine with richer aroma. Much research on mixed fermentations has been dedicated to the $C$. stellata/S. cerevisiae yeasts for their ability to increase the wine's glycerol content and for their particular fructophilic properties (Ciani \& Ferraro, 1998; Ciani \& Fatichenti, 1999), their ability to generate a positive impact on the aromatic complexity of the wine (Scanes et al., 1998) and their ability to reduce the risk of stuck fermentation (Santos et al., 2008). Ciani 
and Ferraro (1998) carried out C. stellata/S. cerevisiae mixed fermentations, following sequential inocula, and produced wines richer in glycerol and succinic acid and with less acetic acid and alcohol than that produced by $S$. cerevisiae alone. Later, Ferraro et al. (2000), in a similar fermentation, produced wines with a lower alcohol content than wines fermented by $S$. cerevisiae alone.

The sensory profiles of wines produced by $C$. stellata/S. cerevisiae mixed fermentation were determined by Soden et al. $(1998,2000)$ and the wine obtained by sequential two-yeast inoculum was enriched with the aroma of banana, flowers and lime, compared to the mono-inoculated $S$. cerevisiae control.

These data suggest that a great complexity and diversity in wines can be obtained with a industrial process when $C$. stellata and $S$. cerevisiae yeasts are selected appropriately. The frequent use of selected yeast strains in winemaking and the tendency to experiment with mixed yeast fermentations require an easy and fast distinction between inoculated yeasts and those that are naturally present in the musts and the possibility of control over the individual growth of various starter yeasts. Currently, different culture agar media are used, either selective or differential, which allows some discrimination between the different microorganism species involved in a wine fermentation. WL nutrient agar (Cavazza et al., 1992; Pallmann et al., 2001; Romancino et al., 2008), YM agar (Soden et al., 2000; Moreira et al., 2005) and YEPD (Ciani et al., 2006; Perez-Nevado et al., 2006) media are generally used to count total yeasts present in an oenological sample. The same media appropriately modified by the addition of an antimycotic or antiseptic (Moreira et al., 2005; Perez-Nevado et al., 2006) are selective and allow the counting of resistant yeasts. Instead, lysine agar (Morris \& Eddy, 1957; Fowell, 1965; Heard \& Fleet, 1986; Cavazza et al., 1992; Soden et al., 2000; Ciani et al., 2006) allows the counting of all the yeasts that are able to use lysine as the only nitrogen source. It follows that two medium types (selective and non-selective) are generally used together, as the information provided is complementary and allows the researchers to trace in some detail the fermentation trend. However, some selected media show an area of application that is restricted to only a few yeast species or genera (Moreira et al., 2005; Perez-Nevado et al., 2006), while when there are $<10^{4}$ Saccharomyces cells/plate on lysine agar the individual cells develop into small colonies that may be mistaken for wild yeast colonies (Morris \& Eddy, 1957; Fowell, 1965). Cavazza and Poznansky (1998) reported that $C$. stellata species grow on WL with a morphological distinction, grow on lysine agar and also on WL differential (WLD) equal to WL medium but containing cycloheximide, a yeast inhibiter active on a large number of yeast species. Thus, given the acquired importance in oenology of the C. stellata/S. cerevisiae mixed fermentations, in this study we have examined a method of investigation that uses the data collected on different culture media, including modified ones, to accurately determine the concentration of the various yeasts present in the analysed sample at each fermentation stage. In particular, the usability of this method was evaluated during the microbiological analysis on plates of a C. stellata strain from the yeast collection of the Sicilian Istituto Regionale della Vite e del Vino (IRVV) together with a commercial $S$. cerevisiae strain.

\section{MATERIALS AND METHODS \\ Yeast strains}

The yeasts used in this study belong to various genera and species: 14 Saccharomyces strains, including some belonging to different collections and most from commercial origins, as shown in Table 1; 42 non-Saccharomyces yeast isolates from the IRVV collection (Romancino et al., 2008), including 14 Candida stellata (Cs1-Cs14), four Hanseniaspora guilliermondii (Hg1-Hg4), five H. uvarum (Hu1-Hu4 and Hu03), three Issatchenkia terricola (It1-It3), four K. thermotolerans (Kt1-Kt4), three Metschnikowia pulcherrima (Mp1-Mp3), three T. delbrueckii (Td1-Td3), three Zygosaccharomyces bailii (Zb1-Zb3) and three Zygoascus hellenicus (Zh1-Zh3); and finally the C. stellata 6714, H. guilliermondii 6796 and $K$. thermotolerans 6232 strains of the University of Perugia's Industrial Yeasts Collection DBVPG (www.agr.unipg.it/dbvpg). The commercial $S$. cerevisiae NDA21 yeast was also selected by the IRVV for the production of high quality wines (Di Maio et al., 2006; Oliva et al., 2006). The isolation and oenological study of the strain $S$. cerevisiae A3-2 also took place in the same selection programme (Di Maio et al., 2009). The Saccharomyces yeasts were cultivated in agar-slant tubes containing Sabouraud dextrose agar (Oxoid), added with $10 \mathrm{~g} / \mathrm{L}$ yeast extract (Oxoid) and maintained at $4^{\circ} \mathrm{C}$. The non-Saccharomyces yeasts were cultivated in agar-slant tubes containing malt agar medium (malt extract $30 \mathrm{~g} / \mathrm{L}$, bacteriological agar $15 \mathrm{~g} / \mathrm{L}$ ) and maintained at $4^{\circ} \mathrm{C}$. All the cultures were renewed periodically.

\section{Plate screening of wine yeasts' cycloheximide resistance on WLD medium}

A loop of yeast fresh cultures, cultivated on YPD agar (yeast extract $10 \mathrm{~g} / \mathrm{L}$, dextrose $20 \mathrm{~g} / \mathrm{L}$, peptone $20 \mathrm{~g} / \mathrm{L}$, agar $20 \mathrm{~g} / \mathrm{L}$ ), was smeared on WL nutrient agar (Oxoid) containing increasing cycloheximide concentrations: $0.5,1.0,2.0,4.0$ and 10.0 $\mathrm{mg} / \mathrm{L}$. The WL medium was prepared following the supplier's instructions, while the WLD medium was prepared as described

\section{TABLE 1}

List of Saccharomyces strains used in the cycloheximide resistance experiment.

\begin{tabular}{lll}
\hline Strain & Yeast & Source \\
\hline A3-2 & S. cerevisiae & IRVV \\
6167 & S. cerevisiae & Diproval \\
L404 & S. cerevisiae & Diproval \\
11719 & S. bayanus & Diproval \\
12233 & S. bayanus & Diproval \\
ICV-D254 & S. cerevisiae & Lallemand \\
EC1118 & S. cerevisiae & Lallemand \\
QD145 & S. cerevisiae & Lallemand \\
Uvaferm43 & S. cerevisiae & Lallemand \\
NDA21 & S. cerevisiae & Biospringer \\
CKS-102 & S. cerevisiae & Biospringer \\
UCLM-S325 & S. cerevisiae & Biospringer \\
Fermol Arome Plus & S. cerevisiae & Pascal Biotech \\
Zymaflore F10 & S. cerevisiae & Laffort \\
\hline
\end{tabular}


by Cavazza et al. (1992), adding cycloheximide to WL medium. The cycloheximide solution was prepared in absolute ethanol $(99.8 \%, \mathrm{v} / \mathrm{v})$ and added to the medium just before the distribution in the Petri dishes. The same medium but without antimycotic was used as a positive control for yeast growth. Yeasts were grown for three to six days at $28^{\circ} \mathrm{C}$. The growth rate with cycloheximide was evaluated by comparing the growth of yeast in the medium with and without antimycotic. Growth on WLD equal to that in WL was considered positive (+) and showed the yeast's ability to grow in the given cycloheximide concentration; no growth was considered negative (-) and showed the yeast's inability to tolerate that concentration of antimycotic. Intermediate growth $(-+,-/-+)$ was indicative of some yeast sensitivity to cycloheximide. The minimum inhibitory concentration (MIC) of cycloheximide was the lowest antimycotic concentration in which the yeast cells did not grow.

This assay was carried out on 14 yeast strains belonging to the Saccharomyces genus from various collections or the industry, and on 45 non-Saccharomyces yeasts belonging to the following species: C. stellata, $H$. guilliermondii, H. uvarum, I. terricola, K. thermotolerans, $M$. pulcherrima, T. delbrueckii, $Z$. bailii and Z. hellenicus (see Table 2). The $S$. cerevisiae NDA21 and $H$. uvarum $H u 03$ strains were used as references for growth in cycloheximide, because the first was sensitive and the second resistant to the antimycotic (Cavazza et al., 1992). All tests were carried out in triplicate.

TABLE 2

Cycloheximide (cyc) resistance of 14 Saccharomyces strains and 45 non-Saccharomyces yeasts. NDA21 and Hu03 (underlined) were used as reference of growth in cyc on WL and WLD with different antimycotic concentrations.

\begin{tabular}{|c|c|c|c|c|c|c|c|c|}
\hline Yeast species & Yeast & $\begin{array}{l}\text { Strain } \\
\text { number }\end{array}$ & $\begin{array}{c}0.0 \\
\mathrm{mg} / \mathrm{L} \\
\mathrm{cyc}\end{array}$ & $\begin{array}{c}0.5 \\
\mathrm{mg} / \mathrm{L} \\
\mathrm{cyc}\end{array}$ & $\begin{array}{c}1.0 \\
\mathrm{mg} / \mathrm{L} \\
\mathrm{cyc}\end{array}$ & $\begin{array}{c}2.0 \\
\mathrm{mg} / \mathrm{L} \\
\mathrm{cyc}\end{array}$ & $\begin{array}{c}4.0 \\
\mathrm{mg} / \mathrm{L} \\
\mathrm{cyc}\end{array}$ & $\begin{array}{c}10.0 \\
\mathrm{mg} / \mathrm{L} \\
\mathrm{cyc}\end{array}$ \\
\hline \multirow[t]{6}{*}{ S. cerevisiae } & $\underline{\mathrm{NDA} 21}, \mathrm{~A} 3-2$ & 2 & + & + & -+ & - & - & - \\
\hline & 6167 & 1 & + & -+ & - & - & - & - \\
\hline & L404 & 1 & + & + & + & - & - & - \\
\hline & ICV-D254 & 1 & + & + & -+ & - & - & - \\
\hline & EC1118 & 1 & + & + & + & -+ & - & - \\
\hline & $\begin{array}{l}\text { QD145, Uvaferm43, CKS-102, } \\
\text { UCLM-S325, Fermol Arome Plus, } \\
\text { Zymaflore F10 }\end{array}$ & 6 & + & + & + & - & - & - \\
\hline \multirow[t]{2}{*}{ S. bayanus } & 11719 & 1 & + & - & - & - & - & - \\
\hline & 12233 & 1 & + & -+ & - & - & - & - \\
\hline H. guilliermondii & 6796, $H g 1$ to $H g 4$ & 5 & + & + & + & + & + & + \\
\hline H. uvarum & $\underline{H u 03}, H u 1$ to $H u 4$ & 5 & + & + & + & + & + & + \\
\hline \multirow[t]{2}{*}{ I. terricola } & It 1, It 2 & 2 & + & + & + & + & - & - \\
\hline & It3 & 1 & + & + & + & - & - & - \\
\hline \multirow[t]{3}{*}{ K. thermotolerans } & 6232 & 1 & + & - & - & - & - & - \\
\hline & $K t 1, K t 2, K t 4$ & 3 & + & + & - & - & - & - \\
\hline & $K t 3$ & 1 & + & -+ & - & - & - & - \\
\hline M. pulcherrima & $M p 1$ to $M p 3$ & 3 & + & + & + & + & -+ & - \\
\hline \multirow[t]{2}{*}{ T. delbrueckii } & $T d 1, T d 3$ & 2 & + & + & - & - & - & - \\
\hline & $T d 2$ & 1 & + & + & + & - & - & - \\
\hline \multirow[t]{2}{*}{ Z. bailii } & $Z b 1$ & 1 & + & + & + & - & - & - \\
\hline & $Z b 2, Z b 3$ & 2 & + & + & + & -+ & - & - \\
\hline Z. hellenicus & $Z h 1$ to $Z h 3$ & 3 & + & + & + & + & + & + \\
\hline \multirow[t]{3}{*}{ C. stellata } & $\begin{array}{l}\text { Cs } 1, \text { Cs } 6, \text { Cs } 10, \text { Cs } 13 \text {, } \\
\text { Cs } 14\end{array}$ & 5 & + & + & + & + & + & + \\
\hline & $\begin{array}{l}C s 2 \text { to } C s 5, C s 7 \text { to } C s 9 \\
C s 11, C s 12\end{array}$ & 9 & + & + & + & + & + & $-/-+$ \\
\hline & 6714 & 1 & + & + & -+ & - & - & - \\
\hline
\end{tabular}


Cycloheximide resistance of pure and mixed plating of $C s 3$ and NDA21 strains on WLD medium

Equal volumes of pure cultures of $C$. stellata $C s 3$ and $S$. cerevisiae NDA21 strains grown in YPD (yeast extract 10 $\mathrm{g} / \mathrm{L}$, dextrose $20 \mathrm{~g} / \mathrm{L}$, peptone $20 \mathrm{~g} / \mathrm{L}$ ) were mixed to obtain a mixture of yeast cells with a concentration corresponding to that of the relative pure culture for each yeast. The mixture and pure cultures were analysed microbiologically following the method described by Cavazza and Poznansky (1998), with some variations: for the three samples, $0.2 \mathrm{~mL}$ of $10^{-4}, 10^{-5}$ and $10^{-6}$ serial dilutions in sterile water were spread on WL and WLD with increasing cycloheximide concentrations of 2.0, 4.0 and $10.0 \mathrm{mg} / \mathrm{L}$. The media were prepared as described in the previous section. The inocula were then incubated at $28^{\circ} \mathrm{C}$ for five to six days and the colony forming units (cfu) grown in the various media were counted. The cfus of $C$. stellata and $S$. cerevisiae yeasts were identified by macroscopic examination of the morphology and colour of the colonies, as described by Cavazza et al. (1992), Cavazza \& Poznansky (1998) and Pallmann et al. (2001). Only data of plates containing between 25 and $250 \mathrm{cfu} /$ plate were considered significant in the counting (Busta et al., 1984). The reported results are averages obtained from three different plating with repeatability within $7.7 \%$ (Cavazza \& Poznansky, 1998).

Selective counting of pure and mixed plating of $\mathrm{Cs} 3$ strain on WLD and lysine agar media

The experiment was carried out as described in the previous section, but spreading dilutions of pure cultures and mixture of the $C s 3$ and NDA21 strains on WL and on WLD media with 2 $\mathrm{mg} / \mathrm{L}$ cycloheximide and on lysine agar, in duplicate. The WL and WLD media were prepared as described in the previous section. The lysine agar (Oxoid) medium was prepared following the supplier's instructions. Only on lysine agar medium, $0.1 \mathrm{~mL}$ of pure culture of the L404 strain (Diproval) grown for $12 \mathrm{~h}$ in $1 \mathrm{~mL}$ of YPD, previously centrifuged (1500 $\mathrm{x} \mathrm{g}, 5 \mathrm{~min}, 4^{\circ} \mathrm{C}$ ) and resuspended in the same volume of sterile water, was widespread on the plates in addition to the $0.2 \mathrm{~mL}$ dilution to achieve a concentration of Saccharomyces higher than $10^{4}$ cells/plate. The inocula were then incubated at $28^{\circ} \mathrm{C}$ and finally the cfus grown in the different media were counted. On the WL and WLD media the colonies of $C$. stellata and $S$. cerevisiae yeasts were identified by macroscopic examination of the morphology and colour, as previously described. The colonies of non-Saccharomyces yeast grown in the lysine agar medium on a monolayer of Saccharomyces, formed by pinpoint colonies, were counted. Only data of plates containing between 25 and $250 \mathrm{cfu} /$ plate were considered significant in the counting (Busta et al., 1984). The reported results are averages obtained from three different plating with repeatability within $7.7 \%$ (Cavazza \& Poznansky, 1998).

\section{Cs3 fermentations in grape must}

Alcoholic fermentations were performed in 2009 vintage from Merlot grapes cultivated in a vineyard situated in Roano county (Palermo, Italy). The grapes were pressed and the must $\left(24.45^{\circ}\right.$ Brix, $\left.\mathrm{pH} 3.55\right)$ was sulphited $(0.05 \mathrm{~g} / \mathrm{L})$. The must was subdivided into two aliquots of $100 \mathrm{~L}$, each one inoculated (6 x $\left.10^{6} \mathrm{cfu} / \mathrm{mL}\right)$ with Cs3 C. stellata liquid culture grown in sterile must $\left(20^{\circ}\right.$ Brix, $\left.\mathrm{pH} 3.20\right)$. Fermentations lasted 13 days

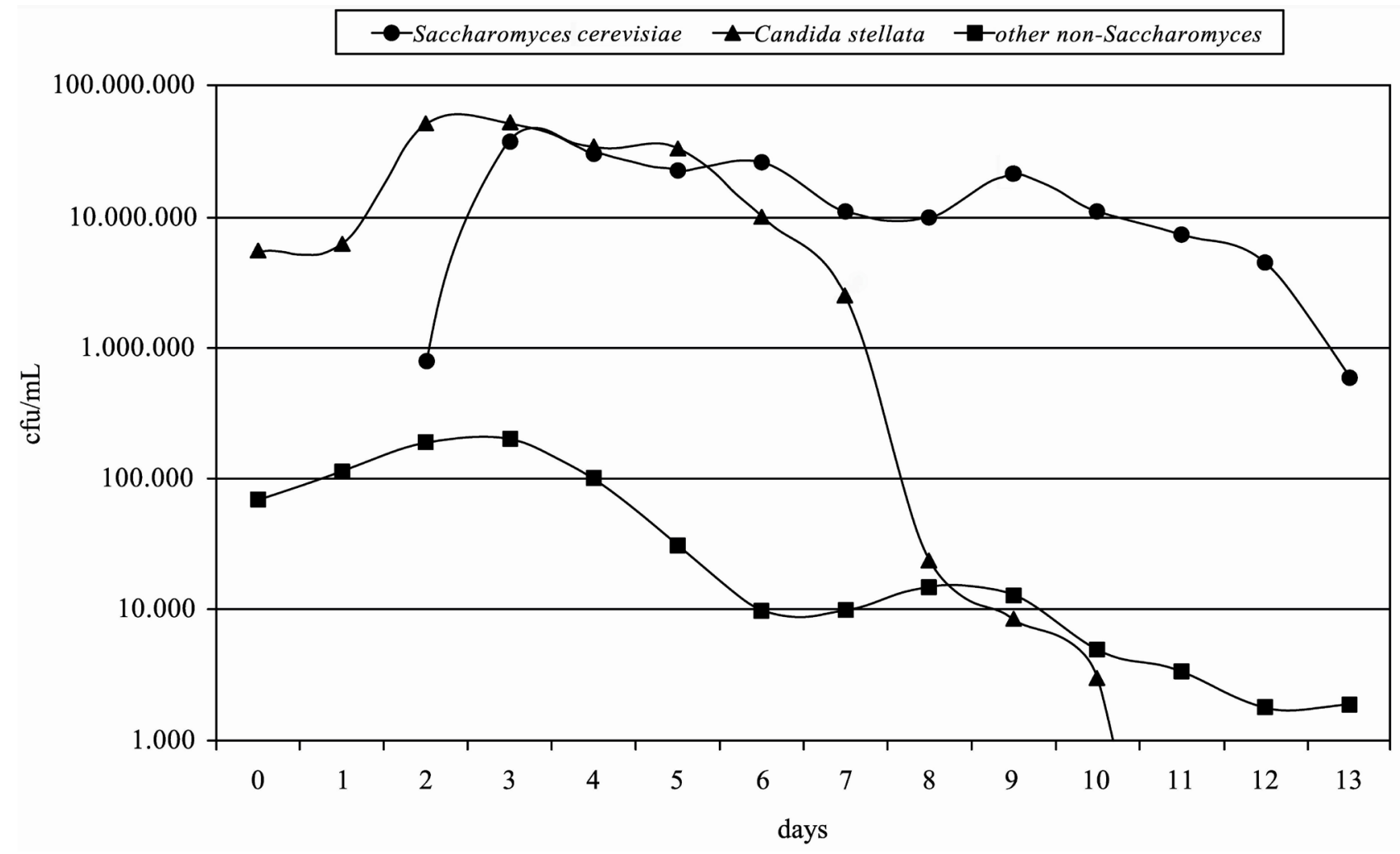

FIGURE 1

Growth curves of Candida stellata, Saccharomyces cerevisiae and other non-Saccharomyces yeasts in a grape must inoculated with $C s 3$ strain. 
TABLE 3

Results of pure and mixed plating of C. stellata Cs3 (IRVV) and $S$. cerevisiae NDA21 (Biospringer) strains spread on WL and WLD with different cycloheximide (cyc) concentrations.

$\begin{array}{ccccc} & c f u / m L & c f u / m L & c f u / m L & c f u / m L \\ \text { Culture } & \text { on } & \text { on } & \text { on } & \text { on }\end{array}$

$0 \mathrm{mg} / \mathrm{L} \mathrm{cyc} 2 \mathrm{mg} / \mathrm{L} \mathrm{cyc} 4 \mathrm{mg} / \mathrm{L} \mathrm{cyc} 10 \mathrm{mg} / \mathrm{L} \mathrm{cyc}$

\begin{tabular}{lcccc}
\hline Cs3 & $150 \times 10^{6}$ & $160 \times 10^{6}$ & $160 \times 10^{6}$ & 0 \\
NDA21 & $50 \times 10^{6}$ & 0 & 0 & 0 \\
Cs3 + NDA21 & $220 \times 10^{6}$ & $150 \times 10^{6}$ & $150 \times 10^{6}$ & 0 \\
\hline
\end{tabular}

TABLE 4

Results of pure and mixed plating of C. stellata Cs3 (IRVV) and $S$. cerevisiae NDA21 (Biospringer) strains spread on lysine agar (LA), WL and WLD with $2 \mathrm{mg} / \mathrm{L}$ cycloheximide (cyc).

\begin{tabular}{lccc}
\hline Yeast strain & $\begin{array}{c}\text { cfu/mL } \\
\text { on } \mathbf{L A}\end{array}$ & $\begin{array}{c}\text { cfu/mL } \\
\text { on } \mathbf{W L}\end{array}$ & $\begin{array}{c}\text { cfu/mL } \\
\text { on WLD } \\
\mathbf{2} \mathbf{~ m g} / \mathbf{L} \text { cyc }\end{array}$ \\
\hline$C s 3$ & $380 \times 10^{6}$ & $360 \times 10^{6}$ & $350 \times 10^{6}$ \\
NDA21 & 0 & $190 \times 10^{6}$ & 0 \\
$C s 3+$ NDA21 & $370 \times 10^{6}$ & $520 \times 10^{6}$ & $350 \times 10^{6}$ \\
\hline
\end{tabular}

at $25^{\circ} \mathrm{C}$. Daily microbiological analyses were performed on WL, WLD (2 mg/L cycloheximide) and lysine agar (Oxoid), as previously described: data referred to in Figure 1 as "other nonSaccharomyces yeasts" were obtained as differences between the counting results in lysine agar and Candida cfus counted on WLD.

The analysis of length of the restriction fragments of polymorphisms of yeast mitochondrial DNA (mtDNA-RFLP) was performed on $50 \mathrm{C}$. stellata cfus from WLD plates on the fifth fermentation day. Yeast DNA was extracted as described by Querol et al. (1992). The total yeast DNA was digested with restriction endonuclease HpaII (Biolabs), according to the supplier's instructions (Pramateftaki et al., 2000). The molecular fragments were separated through horizontal electrophoresis on agarose gel at $0.7 \%(\mathrm{w} / \mathrm{v})$ in $0.5 \mathrm{x}$ TBE (45 mmol Tris base, $45 \mathrm{mmol}$ boric acid, 1 mmol EDTA pH 8.0), using $1 \mathrm{~kb}$ DNA ladder (Promega) as a marker of molecular weight. At the end of the run, the gel was stained with ethidium bromide $(0.5 \mu \mathrm{g} / \mathrm{mL})$ and UV transilluminated. The fluorescent image was acquired using a Gel Doc 2000 system (Bio-Rad Laboratories) running Quantity One software (Bio-Rad Laboratories).

Similar data were obtained in both fermentations. The reported data (Fig. 1) refer only to one.

\section{RESULTS AND DISCUSSION}

\section{Cycloheximide resistance of yeasts}

The aim of this experiment was to evaluate the cycloheximide resistance of various yeast species (Table 2). Although $C$. stellata is generally considered a negative-cycloheximide species, for the 14 strains of $C$. stellata of the IRVV collection our results are partly in agreement with Cavazza and Poznansky (1998), who reported that C. stellata is able to grow up to 4 $\mathrm{mg} / \mathrm{L}$ cycloheximide. However, although these authors advise increasing the amount of cycloheximide in the medium up to 10 $\mathrm{mg} / \mathrm{L}$ to prevent the growth of $C$. stellata, five IRVV strains were able to grow in this antimycotic concentration (Table 2). The $C$. stellata DBVPG 6714 strain, used as a reference from the other collection, shows contrasting behaviour, being sensitive to the lower amount of cycloheximide used in the experiment, equal to $0.5 \mathrm{mg} / \mathrm{L}$.

The results obtained for the 14 Saccharomyces strains analysed in this study, belonging to the $S$. cerevisiae and $S$. bayanus species, confirm the effective sensitivity of these species to the antimycotic (Cavazza et al., 1992; Cavazza \& Poznansky, 1998). The S. bayanus 11719 strain was the most sensitive and was unable to grow even in the lowest antimycotic concentration that was used $(0.5 \mathrm{mg} / \mathrm{L})$. The $S$. cerevisiae EC1118 strain was the only analysed Saccharomyces able to growth in $2 \mathrm{mg} / \mathrm{L}$ cycloheximide, although this growth was reduced. The control strain $S$. cerevisiae NDA21, as expected, was extremely sensitive to the antimycotic, and its growth was already partially inhibited at a concentration of $1 \mathrm{mg} / \mathrm{L}$. The sensitivity to cycloheximide is spread throughout the species, but it is not characteristic of the whole Saccharomyces genus. Deak and Beuchat (1996) report that the species $S$. dairenensis and $S$. exiguus possess some degree of resistance to $100 \mathrm{mg} / \mathrm{L}$ cycloheximide. The MIC of $S$. cerevisiae and $S$. bayanus yeasts used in this experiment therefore corresponds to $2 \mathrm{mg} / \mathrm{L}$ on WL medium, because in such a concentration none of these yeasts is able to grow as in medium without antimycotic.

All the analysed $H$. uvarum and $H$. guilliermondii yeasts are resistant to the highest used cycloheximide concentration, equal to $10 \mathrm{mg} / \mathrm{L}$. The species $H$. guilliermondii, $H$. uvarum and $H$. valbyensis have also been reported to be able to grow in up to $100 \mathrm{mg} / \mathrm{L}$ cycloheximide, while $H$. osmophila is unable to grow at such a high antimycotic concentration (Deak \& Beuchat, 1996). The three analysed Z. hellenicus grew in 10 $\mathrm{mg} / \mathrm{L}$ cycloheximide (Table 2), confirming the resistance of Z. hellenicus to this antimycotic (Prasad et al., 2005). Lower cycloheximide resistance is shown by $M$. pulcherrima, $Z$. bailii and $T$. delbrueckii yeasts, with values that are often different according to the strain, but always lower or equal to 2 $\mathrm{mg} / \mathrm{L}$ : these results agree with those of Cavazza et al. (1992). Nevertheless, we noted partial growth of $M$. pulcherrima in 4 $\mathrm{mg} / \mathrm{L}$ antimycotic. Furthermore, we report similar results for I. terricola and $K$. thermotolerans (Table 2). Thus, the MIC of cycloheximide relating to the analysis of these species is 2 $\mathrm{mg} / \mathrm{L}$, with some reservations only for $M$. pulcherrima. Other Zygosaccharomyces yeasts, such as $Z$. fermentatii and $Z$. florentinus, grow up to $100 \mathrm{mg} / \mathrm{L}$ (Deak \& Beuchat, 1996).

Cycloheximide resistance during microbiological analysis of pure and mixed plating of $C s 3$ and NDA21 strains

The aim of this experiment (Table 3) was to distinguish between $S$. cerevisiae and C. stellata in mixed plating on the basis of their different cycloheximide resistance. The cfu counts of the C. stellata Cs3 and S. cerevisiae NDA21 strains, grown on different media, fully confirmed the data of the previous section. A pure culture of the $C s 3$ strain was able to give comparable 
concentrations in medium without or with 2 and $4 \mathrm{mg} / \mathrm{L}$ cycloheximide (150 to $160 \times 10^{6} \mathrm{cfu} / \mathrm{mL}$ ), showing that these antimycotic concentrations do not cause significant changes in Cs3 growth. On the other hand, just visible and indistinguishable pinpoint cfus grew in $10 \mathrm{mg} / \mathrm{L}$ cycloheximide, but were unable to grow further: growth was therefore considered null. The Cs3 colony morphology was that described by Cavazza and Poznansky (1998), namely "colonies of a pea green colour, which over time become darker at the centre, while the edges are clear, and rising to a dome or umbo", and did not change in shape and/or colour at 2 and $4 \mathrm{mg} / \mathrm{L}$ cycloheximide. However, a delay in the colonies' growth was noted at these cycloheximide concentrations compared to the medium without antimycotic: therefore, the colonies' sizes were different in the various media on the same incubation day and the counting of the colonies required one or two more incubation days than those reported by Cavazza and Poznansky (1998). Extending the incubation time in $10 \mathrm{mg} / \mathrm{L}$ cycloheximide medium showed no late growth of the Cs3 yeast. Conversely, a pure culture of NDA21 strain that was reported to be able to grow on WL "with circular colonies also of considerable diameter and colour varying from cream to green, with domed shape, opaque smooth surface and creamy consistency" (Cavazza et al., 1992), did not grow at any cycloheximide concentration. In the mixed plating of the two yeasts on WL without antimycotic, the $C$. stellata and $S$. cerevisiae colonies were easily distinguished by their different morphology; only C. stellata morphology was found on the 2 and $4 \mathrm{mg} / \mathrm{L}$ cycloheximide media, with an increasing delay in growth at rising antimycotic concentrations.

Selective counting of $C s 3$ yeast during microbiological analysis of pure and mixed plating of $C s 3$ and NDA21 strains In this experiment we used lysine agar, WL and WLD $(2 \mathrm{mg} / \mathrm{L}$ cycloheximide) for collecting as much data as possible about mixtures of $S$. cerevisiae and C. stellata. Table 4 shows the cfu/ plate data obtained by the spreading of $C s 3$ and NDA21 liquid cultures and from a corresponding mixture of the two strains. The pure NDA2 1 culture grew only on WL medium. In contrast, the pure Cs 3 culture grew comparably also in lysine agar and WLD (Table 4), and similar concentrations of $C s 3$ were found when it was plated together with NDA21. However, in the plating of mixed cultures on lysine agar it was necessary to coinoculate a high number of another Saccharomyces strain ( $>$ $10^{4}$ cells/plate), because below this value this species produces colonies that can wrongly be considered as non-Saccharomyces (see Materials and Methods): Candida colonies grown on lysine agar were easily countable on the Saccharomyces monolayer.

\section{Selective counting of $C s 3$ strain during microbiological analysis of wine fermentations}

The use of WL, WLD ( $2 \mathrm{mg} / \mathrm{L}$ cycloheximide) and lysine agar makes it possible to monitor the growth of a strain of C. stellata in the presence of $S$. cerevisiae and other wine yeasts. Figure 1 shows the growth curves of C. stellata, S. cerevisiae and othernonSaccharomyces yeasts in a grape must inoculated with the Cs3 strain. All the non-Saccharomyces yeasts, C. stellata included, were able to grow on lysine agar, but $C$. stellata was identified on WL and WLD by colony morphology. On WL all the other yeasts were also identifiable by different colony morphologies, at least while the $S$. cerevisiae colonies were not so abundant as to make it impossible to recognise any other species. Only genus Hanseniaspora was found on WLD together with C. stellata, confirming that this genus is widespread in the early stages of the spontaneous fermentation of Sicilian grapes (Romancino et al., 2008): their different morphologies allowed their easy and rapid distinction during the counting of the colonies. From the second day, wild Saccharomyces yeasts were found on WL and, as expected, they did not grow on WLD and lysine agar. From the third fermentation day onwards, Saccharomyces yeasts grew increasingly numerously on WL medium, preventing the correct counting of colonies of any other yeast species in this medium, although C. stellata colonies were counted on WLD. From the plating on lysine agar it was possible to collect the data of all the non-Saccharomyces yeasts, even in the early days of fermentation in the presence of a low number of S. cerevisiae cfus, thanks to the Saccharomyces monolayer. mtDNA-RFLP analyses demonstrated the massive presence ( 92 to $100 \%$ ) of the Cs3 strain in the C. stellata population of the fermenting must (data not shown).

\section{CONCLUSIONS}

C. stellata clearly is able to grow at low cycloheximide concentrations, but with important strain-dependent differences: for the $C$. stellata IRVV strains, which showed a higher resistance compared to the available data (Cavazza \& Poznansky, 1998), this phenotypical evidence is a probable sign of their common geographical origin (Romancino et al., 2008). Indeed, studies on $S$. cerevisiae cycloheximide resistance show that mutations in different genetic loci can induce this antimycotic resistance (Del Pozo et al., 1991; Mortimer et al., 1991). These species- and strain-specific differences in cycloheximide resistance make it possible to use an appropriate WLD as tool for monitoring C. stellata strains in all the stages of mixed fermentations. Furthermore, the use of WLD medium has the advantage of distinguishing the cfu morphology of different species and allowing a further check on data obtained from other selective and non-selective media. We demonstrated that it is possible to collect data about a single C. stellata strain in a fermentation with Saccharomyces and non-Saccharomyces yeasts using WL, WLD and lysine agar. Furthermore, this method has the potential to distinguish between $C$. stellata and $K$. thermotolerans, both generally found in grape musts, growing on WL with very similar morphologies (Romancino et al., 2008) but showing very different levels of resistance to cycloheximide. Finally, this method allows the distinction between inoculated and wild $C$. stellata, if coupled with molecular analyses of $C$. stellata strains (Pramateftaki et al., 2000).

\section{LITERATURE CITED}

Busta, F.F., Peterson, E.H., Adams, D.M. \& Johnson, M.G., 1984. Colony count methods. In: Speck, M.L. (ed). Compendium of methods for the microbiological examination of foods. Washington, DC. pp. 62-83.

Cavazza, A. \& Poznansky, E., 1998. Le analisi microbiologiche nel laboratorio enologico. Vignevini 6, 42-53.

Cavazza, A., Grando, M.S. \& Zini, C., 1992. Rilevazione della flora microbica di mosti e vini. Vignevini 9, 17-20.

Ciani, M. \& Fatichenti, F., 1999. Selective sugar consumption by apiculate yeasts. Lett. Appl. Microbiol. 28, 203-206. 
Ciani, M. \& Ferraro, L., 1996. Enhanced glycerol content in wines made with immobilized Candida stellata cells. Appl. Environ. Microbiol. 62, 128-132.

Ciani, M. \& Ferraro, L., 1998. Combined use of immobilized Candida stellata cells and Saccharomyces cerevisiae to improve the quality of wines. J. Appl. Microbiol. 85, 247-254.

Ciani, M., Beco, L. \& Comitini, F., 2006. Fermentation behaviour and metabolic interactions of multistarter wine yeast fermentations. Int. J. Food Microbiol. $108,239-245$.

Clemente-Jimenez, J.M., Mingorance-Carloza, L., Martìnez-Rodrìguez, S., Las Heras-Vàzquez, F.J. \& Rodrìguez-Vico, F., 2004. Molecular characterization and oenological properties of wine yeasts isolated during spontaneous fermentation of six varieties of grape must. Food Microbiol. 21, 149-155.

Deak, T. \& Beuchat, L., 1996. Handbook of food spoilage yeasts. CRC Press, pp. 143-150, Buca Raton, FL.

Del Pozo, L., Abarka, D., Claros, M.G. \& Jimènez, A., 1991. Cycloheximide resistance as a yeast cloning marker. Curr. Genet. 19, 353-358.

Di Maio, S., Polizzotto, G., Notarbartolo, G., Planeta, D., Genovese, S., Gurrieri, C. \& Oliva, D., 2006. Isolamento e caratterizzazione di un nuovo ceppo di lievito di interesse enologico. L'Enologo 11, 93-98.

Di Maio, S., Polizzotto, G., Di Gangi, E., Foresta, G. \& Oliva. D., 2009. Studio delle popolazioni di Saccharomyces indigeni in antiche cantine della Sicilia sud-orientale. www.infowine.com - Rivista Internet di Viticoltura ed Enologia n. $9 / 2,1-13$

Ferraro, L., Fatichenti, F. \& Ciani, M., 2000. Pilot scale vinification process using immobilizer Candida stellata cells and Saccharomyces cerevisiae. Process. Biochem. 35, 1125-1129.

Fleet G.H., 2003. Yeast interactions and wine flavour. Int. J. Food Microbiol. $86,11-22$.

Fowell, R.R., 1965. The identification of wild yeast colonies on Lysine Agar. J. Appl. Bacteriol. 28, 373-383.

Heard, G.M. \& Fleet, G.H., 1986. Evaluation of selective media for enumeration of yeasts during wine fermentation. J. Appl. Bacteriol. 60, 477-481.

Jolly, N.P., Augustyn, O.P.H. \& Pretorius, I.S., 2006. The role and use of nonSaccharomyces yeasts in wine production. S. Afr. J. Enol. Vitic. 27, 15-39.

Moreira, N., Mendes, F., Hogg, T. \& Vasconcelos, I., 2005. Alcohols, esters and heavy sulphur compounds production by pure and mixed cultures of apiculate wine yeasts. Int. J. Food Microbiol. 103, 285-294.

Morris, E.O. \& Eddy, A.A., 1957. Method for the measurement of wild yeast infection in pitching yeast. J. Inst. Brew. 63, 34-43.
Mortimer, R.K., Schild, C.R., Contopoulou, C.R. \& Kans, J.A., 1991. Genetic and physical maps of Saccharomyces cerevisiae. In: Guthrie, C. \& Fink, G.R. (eds). Methods in enzymology: guide to yeast genetics and molecular biology. Academic Press, pp. 827-863, NY.

Oliva, D., Ragusa, M., Capraro, F., Genna, G., Prinzivalli, L., Sparacio, A., Sparla, S. \& Melia, V., 2006. Produzione di vini rossi sperimentali con il ceppo di lievito S. cerevisiae NDA21. L'Enologo 11, 85-92.

Pallmann, C.L., Brown, J.A., Olineka, T.L., Cocolin, L., Mills, D.A. \& Bisson, L.F., 2001. Use of WL medium to profile native flora fermentations. Am. J. Enol. Vitic. 52, 198-203.

Perez-Nevado, F., Albergaria, H., Hogg, T. \& Girio, F., 2006. Cellular death of two non-Saccharomyces wine-related yeasts during mixed fermentations with Saccharomyces cerevisiae. Int. J. Food Microbiol. 108, 336-345.

Pramateftaki, P.V., Lanardis, P. \& Typas, M.A., 2000. Molecular identification of wine yeasts at species or strain level: a case study with strains from two vinegrowing areas of Greece. J. Appl. Microbiol. 89, 236-248.

Prasad, G.S., Maylraj, S., Nitu, S., Vijeyta, S., Kakoli, B. \& Banwari, L., 2005. Candida digboiensis sp. nov., a novel anamorphic yeast species from an acidic tar sludge-contaminated oilfield. Int. J. Syst. Evol. Microbiol. 55, 967-972.

Querol, A., Barrio, E., Huerta, T. \& Ramon, D., 1992. Molecular monitoring of wine fermentation conducted by active dry yeast strains. Appl. Environ. Microbiol. 58, 2948-2953.

Romancino, D., Di Maio, S., Muriella, R. \& Oliva, D., 2008. Analysis of non Saccharomyces yeast populations isolated from grape must from Sicily (Italy). J. Appl. Microbiol. 6, 2248-2254.

Romano, P., Fiore, C., Paraggio, M., Caruso, M. \& Capece, A., 2003. Function of yeast species and strains in wine flavour. Int. J. Food Microbiol. 86, 169-180.

Santos, J., Sousa, M.J., Cardoso, H., Inàcio, J., Silva, S., Spencer-Martins, I. \& Leão, C., 2008. Ethanol tolerance of sugar transport and the rectification of stuck wine fermentations. Microbiology 154, 422-430.

Scanes, K.T., Hohmann, S. \& Prior, B.A., 1998. Glycerol production by the yeast Saccharomyces cerevisiae and its relevance to wine: a review. S. Afr. J. Enol. Vitic. 19, 15-21.

Soden, A., Francis, I.L., Gockowiak, H., Lee, T.H. \& Henschke, P.A., 1998. The use of non-Saccharomyces yeasts in winemaking. In: Blair, R.J., Sas, A.N., Hayes, P.F. \& Høj, P.B. (eds). Proc. $10^{\text {th }}$ Aust. Wine. Ind. Tech. Conf., August 1998, Sydney, Australia. pp. $166-171$.

Soden, A., Francis, I.L., Oakey, H. \& Henschke, P.A., 2000. Effects of cofermentation with Candida stellata and Saccharomyces cerevisiae on the aroma and composition of Chardonnay wine. Aust. J. Grape Wine Res. 6, 21-30. 